\title{
Two-anticoloring of planar and related graphs
}

\author{
Daniel Berend ${ }^{1}$ and Ephraim Korach ${ }^{2}$ and Shira Zucker ${ }^{3 \dagger}$ \\ ${ }^{1}$ Departments of Mathematics and Computer Science, Ben-Gurion University, Beer-Sheva 84105, Israel. \\ ${ }^{2}$ Department of Industrial Engineering and Management, Ben-Gurion University, Beer-Sheva 84105, Israel. \\ ${ }^{3}$ Department of Computer Science, Ben-Gurion University, Beer-Sheva 84105, Israel.
}

\begin{abstract}
An anticoloring of a graph is a coloring of some of the vertices, such that no two adjacent vertices are colored in distinct colors. We deal with the anticoloring problem with two colors for planar graphs, and, using Lipton and Tarjan's separation algorithm, provide an algorithm with some bound on the error. In the particular cases of graphs which are strong products of two paths or two cycles, we provide an explicit optimal solution.
\end{abstract}

Keywords: Graph, algorithm, combinatorial optimization, graph anticoloring, separation

\section{Introduction}

An anticoloring of a graph is a coloring of some of the vertices, such that no two adjacent vertices are colored in distinct colors. In the basic anticoloring problem we are given an undirected graph $G$ and positive integers $B_{1}, \ldots, B_{k}$, and have to determine whether there exists an anticoloring of $G$ such that $B_{j}$ vertices are colored in color $j, j=1, \ldots, k$.

The anticoloring problem with $k=2$ is the Black-and-White Coloring (BWC) problem. We usually refer to the optimization version of the BWC problem, in which we are given a graph $G$ and a positive integer $B$, and have to color $B$ of the vertices in black, so that there will remain as many vertices as possible which are non-adjacent to any of the $B$ vertices. (These latter vertices are to be colored in white.) We denote by $W$ the maximum possible number of such vertices.

The problem was originated by Berge, who suggested a special instance (see [5]): Given positive integers $n$ and $B$, place $B$ black and $W$ white queens on an $n \times n$ chessboard, so that no black queen and white queen attack each other, and with $W$ as large as possible. The complexity of the queens problem is still open.

The BWC problem has been introduced and proved to be $N P$-complete by Hansen, Hertz and Quinodoz [5]. In the same paper, an $O\left(n^{3}\right)$ algorithm for trees was given. Kobler, Korach and Hertz [1] gave a polynomial algorithm for partial $k$-trees with fixed $k$. Yahalom [6] investigated an analogous problem to that suggested by Berge, using rooks instead of queens. She gave a sub-linear algorithm to this problem. For special cases, in which the ratio between the sides of the board is an integer or close to an integer, she derived an explicit formula for the optimal solution.

Given a graph $G=(V, E)$, a black-white coloring $(B W C)$ of $G$ is a function

$$
C: V \rightarrow\{\text { black, white, uncolored }\}
$$

such that there is no edge between a black and a white vertex.

Note that $C$ is uniquely determined by the set of vertices colored in black, in the following sense. All vertices, which are adjacent to some black vertex, must be left uncolored. On the other hand, all other vertices may be colored in white. Without loss of generality, we shall assume that this is the case. Thus, when referring to a coloring $C$, it suffices to refer to its black vertices. The problem of maximizing $W$ is equivalent to the problem of minimizing the set of uncolored vertices of the coloring.

In Section 2 we discuss the separation problem, which is a similar problem to BWC. Using the separation algorithm of Lipton and Tarjan's, we provide an algorithm with some bound on the error for the anticoloring of planar graphs. The graphs we discuss in Section 3 are strong product of two graphs: the toroidal grid - of two simple cycles, and the non-toroidal grid - of two simple paths. Section 4 gives a sketch of the proof of our results on non-toroidal grids.

${ }^{\dagger}$ Partially supported by the Lynn and William Frankel Center for Computer Sciences. 


\section{The Separation Problem}

The separation of graphs is a similar problem to anticoloring. Lipton and Tarjan [3] obtained the following result for separation of planar graphs.

Theorem 2.1 [3] Let $G$ be any n-vertex planar graph. The vertices of $G$ can be partitioned into three sets $T_{1}, T_{2}, C$, such that no edge joins a vertex in $T_{1}$ with a vertex in $T_{2}$, neither $T_{1}$ nor $T_{2}$ contains more than $\frac{2}{3} n$ vertices and $C$ contains no more than $2 \sqrt{2} \sqrt{n}$ vertices.

The proof of the theorem is constructive and provides a linear time algorithm for finding a separation satisfying the required properties. We use this algorithm to find a "good" anticoloring of planar graphs. The algorithm of Lipton and Tarjan will be related to as Algorithm LT. Without loss of generality, we assume that in the theorem we have $\left|T_{1}\right| \geq\left|T_{2}\right|$.

\section{Problem 2.1 ColorGraph}

Input: A planar graph $G=(V, E)$ and an integer $B \leq|V|$.

Output: An optimal anticoloring $C$ of $G$ with $B$ black vertices.

Algorithm 1 provides a heuristic for Problem 2.1 with an explicit upper bound on the deviation from the optimum.

Theorem 2.2 Given a planar graph $G$ and a number $B \in\{1,2, \ldots,|V|\}$, Algorithm 1 finds an anticoloring with $B$ black vertices and $W \geq n-B-O(\min \{\sqrt{n}, B\})$ white vertices. The complexity of Algorithm 1 is $O(n)$.

Remark 2.3 The proof of Theorem 2.2 provides an upper bound of $6 \sqrt{2}(1+\sqrt{2 / 3}) \sqrt{n}$ on the number of uncolored vertices. This bound depends on the bound in Theorem 2.1, and can be reduced, theoretically, by using the separation theorem of [4]. (However, the result of [4] does not provide an algorithm for the improved separation.) Similarly, it can be reduced, theoretically, by using the separation theorem of [2] for maximal planar graphs.

At each round of the while loop, $n^{\prime}$ is decreased by at least $\frac{1}{3}$ of its size. Therefore, we have immediately Lemma 2.4 The while loop in Algorithm 1 is executed at most $\log _{3 / 2} n$ times.

Proof of Theorem 2.2: Obviously, Algorithm 1 finds an anticoloring of $G$ with $B$ black vertices.

If $B<\sqrt{n}$, the algorithm takes $B$ vertices with relatively small degrees. (For example, we may take $B$ vertices with degree at most 12 . This can be done because in a planar graph there are at most $3 n-6$ edges which implies that the average degree is at most 6 . Hence there are at least $\frac{n}{2}$ vertices with degree at most 12. Since $B<\sqrt{n} \leq \frac{n}{2}$, there are enough vertices to take.) This gives $W \geq n-B-O(B)$, and can be done in linear time. (Moreover, on the average this takes $O(B)$ time. )

Otherwise, denote by $n_{i}^{\prime}$ the number of vertices in the graph $G^{\prime}$ of the $i$-th call to Algorithm LT. Denote by $C_{i}$ the uncolored vertices left after the $i$-th call to Algorithm LT. The uncolored vertices we find for the original graph $G$ are contained in $\bigcup_{i=1}^{t} C_{i}$, where $t$ is the number of times Algorithm LT is being called. Obviously,

$$
\left|C_{i}\right| \leq 2 \sqrt{2} \sqrt{n_{i}^{\prime}} .
$$

We know that

$$
n_{i+1}^{\prime} \leq \frac{2}{3} n_{i}^{\prime}
$$

and therefore,

$$
\left|\bigcup_{i=1}^{t} C_{i}\right| \leq 2 \sqrt{2} \sqrt{n} \sum_{i=0}^{\infty} \sqrt{(2 / 3)^{i}}=\frac{2 \sqrt{2} \sqrt{n}}{1-\sqrt{\frac{2}{3}}}=6 \sqrt{2} \sqrt{n}(1+\sqrt{2 / 3})=O(\sqrt{n}) .
$$

The complexity of Algorithm LT is known to be $O(n)$. By Lemma 2.4, we have $t \leq \log _{3 / 2} n$, so that the complexity of Algorithm 1 is

$$
O\left(\sum_{i=0}^{\log _{3 / 2} n} n \cdot\left(\frac{2}{3}\right)^{i}\right)=O(n)
$$


Algorithm 1: Anticoloring of a planar graph.

Input: A planar graph $G=(V, E)$ and a number $B \in\{1,2, \ldots,|V|\}$.

Output: An anticoloring of $G$ with $B$ vertices and $O(\sqrt{n})$ uncolored vertices.

$\operatorname{PLANAR}(G, B)$

(1) $\quad$ if $(B<\sqrt{n})$

(2) choose any B vertices with relatively small degrees

(3) else

$$
\begin{aligned}
& G^{\prime} \leftarrow G / / \text { current subgraph } \\
& n^{\prime} \leftarrow n / / \text { number of vertices of the current subgraph } \\
& B^{\prime} \leftarrow B / / \text { number of vertices still to be colored black } \\
& S \leftarrow \emptyset / / \text { set of black vertices } \\
& T_{1}, T_{2} \leftarrow \emptyset / / \text { subgraphs created by last call to LT } \\
& \text { while } B^{\prime} \leq n^{\prime}-6 \sqrt{2} \sqrt{n^{\prime}}(1+\sqrt{2 / 3})
\end{aligned}
$$$$
\left(T_{1}, T_{2}\right) \leftarrow \operatorname{LT}\left(G^{\prime}\right)
$$$$
n_{1} \leftarrow\left|T_{1}\right|
$$$$
n_{2} \leftarrow\left|T_{2}\right| / / \text { recall that } n_{2} \leq n_{1}
$$$$
\text { bool }_{1} \leftarrow\left(n_{1}>B^{\prime}\right)
$$$$
\text { bool }_{2} \leftarrow\left(n_{2}>B^{\prime}\right)
$$$$
\text { switch }\left(\text { bool }_{1}, \text { bool }_{2}\right)
$$$$
\text { case (true, true) }
$$$$
\text { if }\left(\frac{2}{3} n_{1} \leq B^{\prime}\right)
$$$$
G^{\prime} \leftarrow T_{1} / / G^{\prime} \text { is the subgraph still to be divided }
$$$$
n^{\prime} \leftarrow n_{1}
$$

end while

pick arbitrarily $B^{\prime}$ vertices from $G^{\prime}$ and add them to $S$ return $S$

\section{The Kings Problem}

We now focus on a special instance of the $B W C$ problem. As mentioned above, Berge suggested the problem of placing non-attacking black and white queens on a chessboard. We consider the analogous problem with kings instead of queens.

Problem 3.1 Given positive integers $m, n$ and B, place B black and $W$ white kings on an $m \times n$ chessboard, so that no black king and white king attack each other, and with $W$ as large as possible.

Note that Problem 3.1 is in fact the BWC problem for the strong product $P_{m} \otimes P_{n}$ of two simple paths.

When discussing an optimal coloring, we will color a square region. Therefore, in order to distinguish between the colored square region and the squares of the board, we will refer to the latter ones as vertices of a general graph. In the sequel we shall assume without loss of generality that $m \geq n$. The rows of the board are enumerated, from top to bottom, by the numbers $1,2, \ldots, n$, and the columns, from left to right, by the numbers $1,2, \ldots, m$. The vertex at row $i$ and column $j$ is denoted by $(i, j)$.

We now provide an algorithm for coloring the vertices of an $m \times n$ board, solving our optimality problem. It turns out that the optimal coloring behaves differently depending on the size of $B$. For small 
$B$ (up to $n^{2} / 4$ approximately), an optimal coloring may be obtained by coloring an almost square region. For intermediate $B$ (from $n^{2} / 4$ up to $m n-n^{2} / 4$ approximately), an optimal coloring may be obtained by coloring an almost rectangular region, consisting of about $B / n$ adjacent full columns. For large $B$, an optimal coloring may be obtained by coloring almost the complement of a square. The black vertices of the above three colorings should be placed at one end of the board. More formally, we shall prove

Theorem 3.1 Consider Problem 3.1, where $m \geq n \geq 1$. An optimal solution may be constructed, depending on the size of $B$, as follows:

1. $B \leq\left(\frac{n-1}{2}\right)^{2}$ :

Let:

$$
a=\lceil\sqrt{B}\rceil \text { and } r=B-(a-1)\left\lceil\frac{B}{a}\right\rceil .
$$

Color in black the set $\{1, \ldots, a-1\} \times\left\{1, \ldots,\left\lceil\frac{B}{a}\right\rceil\right\} \cup\{a\} \times\{1, \ldots, r\}$. (See Fig. 1.a.)

2. $\left(\frac{n-1}{2}\right)^{2}<B \leq m n-\left(\frac{n+1}{2}\right)^{2}$ :

Let:

$$
a=\left\lceil\frac{B}{n}\right\rceil \text { and } r=B-(a-1) n .
$$

Color in black the set $\{1, \ldots, n\} \times\{1, \ldots, a-1\} \cup\{1, \ldots, r\} \times\{a\}$. (See Fig. 1.b.)

3. $m n-\left(\frac{n+1}{2}\right)^{2}<B$ :

Let

$$
a=\lceil\sqrt{m n-B}\rceil \text { and } r=a \cdot\left\lceil\frac{m n-B}{a}\right\rceil-(m n-B) .
$$

Color in black the set $\{a+1, \ldots, n\} \times\{1, \ldots, m\} \cup\{1, \ldots, n\} \times\left\{\left\lceil\frac{m n-B}{a}\right\rceil+1, \ldots, m\right\} \cup\{a\} \times$ $\left\{\left\lceil\frac{m n-B}{a}\right\rceil-r+1, \ldots,\left\lceil\frac{m n-B}{a}\right\rceil\right\}$. (See Fig. 1.c.)
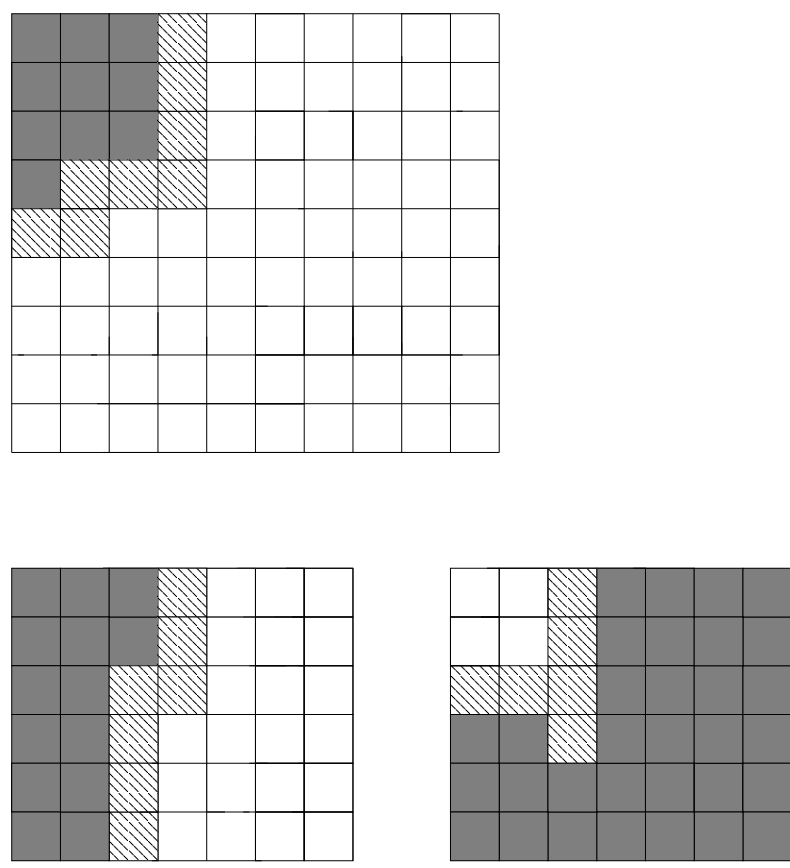

Fig. 1: (a) Small B (b) Intermediate B (c) Large B

The theorem enables us to provide an explicit formula, in case where $n \geq 4$, for $W_{\mathrm{opt}}$ - the largest possible $W$-in terms of $B$, as follows: 


$$
W_{\mathrm{opt}}=m n-B- \begin{cases}\lceil 2 \sqrt{B}\rceil+1, & 1 \leq B \leq\left(\frac{n-1}{2}\right)^{2}, \\ n+S^{\prime}, & \left(\frac{n-1}{2}\right)^{2}<B \leq m n-\left(\frac{n+1}{2}\right)^{2}, \\ \lceil\sqrt{4(m n-B)-2}\rceil-1, & m n-\left(\frac{n+1}{2}\right)^{2}<B \leq m n-4, \\ m n-B, & m n-4<B \leq m n\end{cases}
$$

where

$$
S^{\prime}= \begin{cases}0, & B \equiv 0(\bmod n) \\ 1, & B \not \equiv 0(\bmod n) .\end{cases}
$$

Now consider the case where our $m \times n$ board is toroidal. We have a very similar result for this graph.

Theorem 3.2 Consider Problem 3.1, where $m \geq n \geq 1$ and the board is toroidal. An optimal solution may be constructed, depending on the size of $B$, as follows:

1. $B \leq\left(\frac{n}{2}-1\right)^{2}$ :

Let

$$
a=\lceil\sqrt{B}\rceil \text { and } r=B-(a-1)\left\lceil\frac{B}{a}\right\rceil .
$$

Color in black the set $\{1, \ldots, a-1\} \times\left\{1, \ldots,\left\lceil\frac{B}{a}\right\rceil\right\} \cup\{a\} \times\{1, \ldots, r\}$.

2. $\left(\frac{n}{2}-1\right)^{2}<B \leq m n-\left(\frac{n}{2}+1\right)^{2}-2$ :

Let

$$
a=\left\lceil\frac{B}{n}\right\rceil \text { and } r=B-(a-1) n .
$$

Color in black the set $\{1, \ldots, n\} \times\{1, \ldots, a-1\} \cup\{1, \ldots, r\} \times\{a\}$.

3. $m n-\left(\frac{n}{2}+1\right)^{2}-2<B$ :

Let

$$
a=\lceil\sqrt{m n-B}\rceil \text { and } r=a \cdot\left\lceil\frac{m n-B}{a}\right\rceil-(m n-B) .
$$

Color in black the set $\{a+1, \ldots, n\} \times\{1, \ldots, m\} \cup\{1, \ldots, n\} \times\left\{\left\lceil\frac{m n-B}{a}\right\rceil+1, \ldots, m\right\} \cup\{a\} \times$ $\{1, \ldots, r\}$.

The theorem enables us to provide an explicit formula for $W_{\text {opt }}$ in case where $n \geq 4$, namely,

$$
W_{\mathrm{opt}}=m n-B- \begin{cases}2\lceil 2 \sqrt{B}\rceil+4, & 1 \leq B \leq\left(\frac{n}{2}-1\right)^{2}, \\ 2 n+\mathrm{S}, & \left(\frac{n}{2}-1\right)^{2}<B \leq m n-\left(\frac{n}{2}+1\right)^{2}-2, \\ \lceil 2 \sqrt{4(m n-B)-2}\rceil-4, & m n-\left(\frac{n}{2}+1\right)^{2}-2<B \leq m n-9, \\ m n-B, & m n-9<B \leq m n,\end{cases}
$$

where

$$
S=1-\operatorname{sgn}\left(\frac{B+1}{n}-\left\lceil\frac{B}{n}\right\rceil\right)= \begin{cases}0, & B \equiv 0(\bmod n), \\ 1, & B \equiv-1(\bmod n), \\ 2, & B \neq 0,-1(\bmod n) .\end{cases}
$$

\section{Sketch of the Proof of Theorem 3.2}

The proofs of Theorems 3.1 and 3.2 are very similar. We give a sketch of the proof only for the second theorem.

Denote by $N(C)$ the number of uncolored vertices in a coloring $C$. Recall that only neighbors of black vertices are left uncolored.

Denote by $C_{0}$ the coloring described in the theorem.

For any coloring $C$, we make a series of changes of two kinds:

1. Moving some of the black vertices of $C$ without enlarging the number of uncolored vertices. 
2. Adding black vertices without enlarging the number of uncolored vertices.

By the end of these stages, assuming, by negation, that we started from a coloring $C$ which is strictly better than $C_{0}$ (i.e. $N(C)<N\left(C_{0}\right)$ ), we arrive at a coloring $C^{\prime}$ such that $N(C) \geq N\left(C^{\prime}\right) \geq N\left(C_{0}\right)$. Note that $C^{\prime}$ may well contain more black vertices than $C$ and $C_{0}$ do, but in any case the resulting inequality $N(C) \geq N\left(C_{0}\right)$ yields a contradiction.

An example of the first kind of changes is given in

Lemma 4.1 Suppose a coloring $C$ contains three empty columns (rows, resp.), of which two are adjacent, say columns $j, m-1, m$ (rows $i, n-1, n$, resp.). The coloring $C^{\prime}$, obtained from $C$ by moving columns $j+1, j+2, \ldots, m-2$ (rows $i+1, i+2, \ldots, n-2$, resp.) one place to the left (up, resp.) and column $j$ immediately to their right (row $i$ immediately under them, resp.), satisfies $N\left(C^{\prime}\right) \leq N(C)$. (See Fig. 2.)

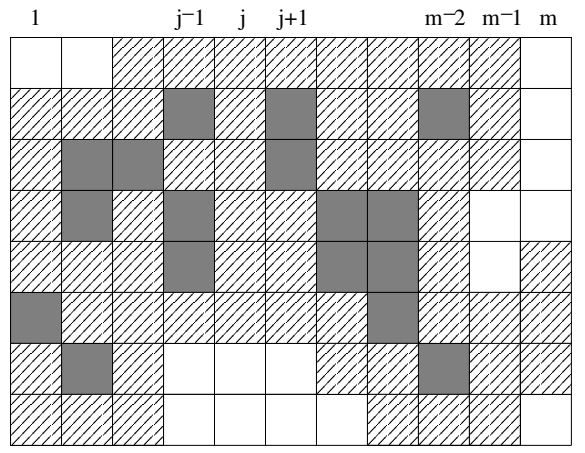

$\mathrm{C}$ with $\mathrm{B}=17$ and $\mathrm{N}(\mathrm{C})=55$

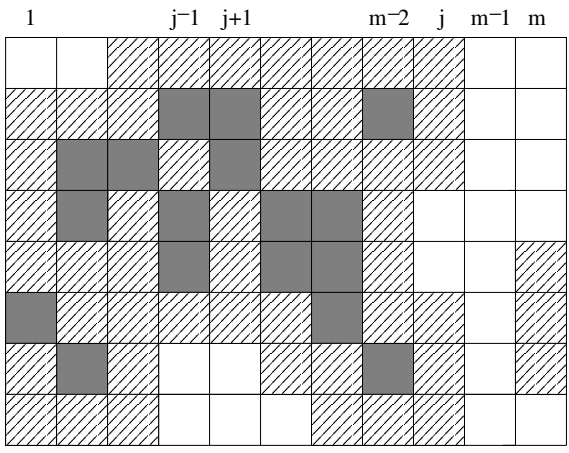

$\mathrm{C}^{\prime}$ with $\mathrm{B}=17$ and $\mathrm{N}\left(\mathrm{C}^{\prime}\right)=49$

Fig. 2: The effect of putting the empty columns together

Proof: We shall prove the lemma for columns. The same proof applies to rows.

Denote by $N_{k}(C)$ and $N_{k}\left(C^{\prime}\right)$ the number of uncolored vertices in column $k$ of $C$ and column $k$ of $C^{\prime}$, respectively. Let us count the uncolored vertices of $C^{\prime}$. Clearly, if $1 \leq k \leq m$, and $k \neq j-1, j, j+$ $1, m-1$, then $N_{k}\left(C^{\prime}\right)=N_{k}(C)$.

It is easy to show that $N_{m-1}\left(C^{\prime}\right)=0$ and $N_{j}\left(C^{\prime}\right)=N_{m-1}(C)$. All uncolored vertices in columns $j-1$ and $j+1$ of $C$ remain uncolored in $C^{\prime}$. Furthermore, some of the white vertices in those two columns may become uncolored in $C^{\prime}$. If $(i, j-1)$ or $(i, j+1)$ is one of those new uncolored vertices, then $(i, j)$ was uncolored in $C$. Likewise, only one of the two vertices $(i, j-1)$ and $(i, j+1)$ might be a new uncolored vertex. Thus, the number of all the new uncolored vertices in both columns $j-1$ and $j+1$ together, is at most $N_{j}(C)$. Therefore,

$$
N_{j-1}\left(C^{\prime}\right)+N_{j+1}\left(C^{\prime}\right) \leq N_{j-1}(C)+N_{j}(C)+N_{j+1}(C),
$$

and hence

$$
\begin{aligned}
N_{j-1}\left(C^{\prime}\right)+N_{j}\left(C^{\prime}\right)+ & N_{j+1}\left(C^{\prime}\right)+N_{m-1}\left(C^{\prime}\right) \\
& \leq N_{j-1}(C)+N_{j}(C)+N_{j+1}(C)+N_{m-1}(C),
\end{aligned}
$$

which implies the required inequality.

An example of the second kind of changes is given in

Lemma 4.2 Suppose a coloring $C$ contains (among others) two black vertices $\left(i_{1}, j_{1}\right)$ and $\left(i_{2}, j_{2}\right)$. If $\left(i_{1}, j_{1}\right)$ and $\left(i_{2}, j_{2}\right)$ may be reached from each other by a chess knight move, i.e., $\left(i_{2}, j_{2}\right)=\left(i_{1} \pm\right.$ $\left.2(\bmod n), j_{1} \pm 1(\bmod m)\right)$ or $\left(i_{2}, j_{2}\right)=\left(i_{1} \pm 1(\bmod n), j_{1} \pm 2(\bmod m)\right)$, then, by coloring in black one of their two common neighbors (if it is not already black), we obtain a coloring $C^{\prime}$ with $N\left(C^{\prime}\right) \leq N(C)$.

The proof is straightforward (Fig. 3). For example, take the third case with $\left(i_{1}, j_{1}\right)$ and $\left(i_{2}, j_{2}\right)=$ $\left(i_{1}+1(\bmod n), j_{1}+2(\bmod m)\right)$ colored in black. By coloring in black one of its two common neighbors, for example $\left(i_{1}+1(\bmod n), j_{1}+1(\bmod m)\right)$, the only vertex which may change from white to uncolored is $\left(i_{1}+2(\bmod n), j_{1}\right)$. However, by coloring $\left(i_{1}+1(\bmod n), j_{1}+1(\bmod m)\right)$ we subtract one uncolored vertex. Thus, $N\left(C^{\prime}\right)=N(C)-1$ or $N\left(C^{\prime}\right)=N(C)$. 

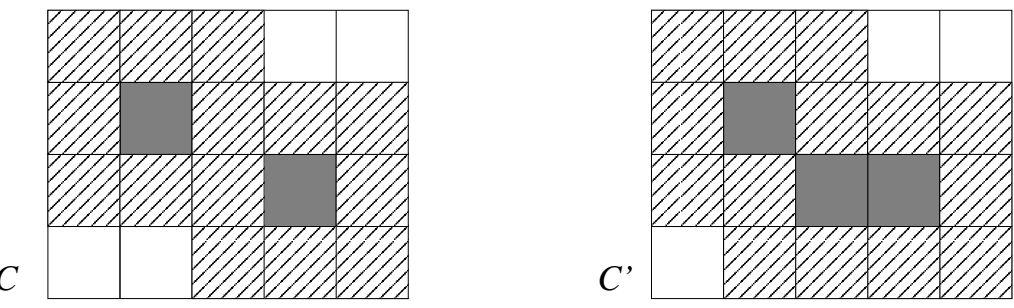

Fig. 3: The effect of changes in Lemma 4.2

\section{References}

[1] E. Korach D. Kobler and A. Hertz. On black-and-white colorings, Anticolorings and Extensions. preprint.

[2] H. Djidjev and S. Venkatesan. Reduced constants for simple cycle graph separation, 1997.

[3] R. J. Lipton and R. E. Tarjan. A separator theorem for planar graphs, 1979.

[4] P. Seymour N. Alon and R. Thomas. Planar separators, 1994.

[5] A. Hertz P. Hansen and N. Quinodoz. Splitting trees, 1997.

[6] O. Yahalom. Anticoloring problems on graphs, 2001. M.Sc Thesis, Ben Gurion University. 
